\title{
Inhibitory effects of 3-bromopyruvate in human nasopharyngeal carcinoma cells
}

\author{
XUE ZOU $^{1 *}$, MENGXIAO ZHANG $^{1 *}$, YIMING SUN ${ }^{1}$, SURONG ZHAO $^{1}$, YINGMEI WEI $^{1}$, \\ XUDONG ZHANG ${ }^{2}$, CHENCHEN JIANG ${ }^{2}$ and HAO LIU ${ }^{1}$ \\ ${ }^{1}$ Faculty of Pharmacy, Bengbu Medical College, Bengbu, Anhui 233004, P.R. China; \\ ${ }^{2}$ Priority Research Center for Cancer Research, University of Newcastle, Callaghan, NSW 2308, Australia
}

Received April 22, 2015; Accepted July 2, 2015

DOI: $10.3892 / o r .2015 .4147$

\begin{abstract}
Tumor cells depend on aerobic glycolysis for adenosine triphosphate (ATP) production, which is therefore targeted by therapeutic agents. The compound 3-bromopyruvate (3-BrPA), a strong alkylating agent and hexokinase inhibitor, inhibits tumor cell glycolysis and the production of ATP, causing apoptosis. 3-BrPA induces apoptosis of nasopharyngeal carcinoma (NPC) cell lines HNE1 and CNE-2Z, which may be related to its molecular mechanisms. In the present study, we investigated the effects of 3-BrPA on the viability, reactive oxygen species (ROS), apoptosis and other types of programmed cell death in NPC cells in vitro and in vivo. PI staining showed significant apoptosis in NPC cells accompanied by the overproduction of ROS and downregulation of mitochondrial membrane potential (MMP, $\triangle \Psi \mathrm{m}$ ) by 3-BrPA. However, the ROS scavenger N-acetyl-L-cysteine (NAC) significantly reduced 3-BrPA-induced apoptosis by decreasing ROS and facilitating the recovery of MMP. We elucidated the molecular mechanisms underlying 3-BrPA activity and found that it caused mitochondrial dysfunction and ROS production, leading to necroptosis of NPC cells. We investigated the effects of the caspase inhibitor z-VAD-fmk, which inhibits apoptosis but promotes death domain receptor (DR)-induced NPC cell necrosis. Necrostatin-1 (Nec-1) inhibits necroptosis, apparently via a DR signaling pathway and thus abrogates the effects of z-VAD-fmk. In addition, we demonstrated
\end{abstract}

Correspondence to: Dr Chenchen Jiang, Priority Research Center for Cancer Research, University of Newcastle, Callaghan, NSW 2308, Australia

E-mail: chenchen_jiang@yahoo.com.cn

Dr Hao Liu, Faculty of Pharmacy, Bengbu Medical College, Bengbu, Anhui 233004, P.R. China

E-mail: liuhao6886@foxmail.com

*Contributed equally

Key words: 3-bromopyruvate, nasopharyngeal carcinoma cells, apoptosis, necroptosis, reactive oxygen species the effective attenuation of 3-BrPA-induced necrotic cell death by Nec-1. Finally, animal studies proved that 3-BrPA exhibited significant antitumor activity in nude mice. The present study is the first demonstration of 3-BrPA-induced non-apoptotic necroptosis and ROS generation in NPC cells and provides potential strategies for developing agents against apoptosis-resistant cancers.

\section{Introduction}

Nasopharyngeal carcinoma (NPC), a malignant tumor, is highly prevalent in the Southeast Asian population (1). The pathological analysis of these tumors mostly shows poorly differentiated squamous cells and therefore, the preferred method of treatment is radiation therapy. However, complications associated with radiation therapy are common; the advantage offered by this treatment is only limited to early in situ non-metastatic carcinoma and the 5-year survival rate is quite low. Therefore, the development of new chemotherapeutic alternatives with maximum efficacy and low toxicity against NPC is necessary.

The development of malignant tumors such as NPC is associated with tumor cell growth and the growth of new blood vessels, which provide nutrients and oxygen capacity since the existing vessels cannot meet the needs of the growing tumor. Tumor cells require enhanced glycolysis to maintain strong autonomous and physiological activities $(2,3)$. The enhanced glycolysis leads to reduced mitochondrial respiration in tumor cells, which ultimately depends on aerobic glycolysis for adenosine triphosphate (ATP) release as a priority source of energy to meet their needs. Furthermore, even with an adequate oxygen supply, enhanced glycolysis still weakens cellular aerobic oxidation and enhances sugar fermentation (4). In order to elucidate the role of glycolysis in ATP production in malignant and normal cells, we first determined the intracellular levels of ATP after treatment with a potent inhibitor of glycolysis in tumor cells.

It is well known that this cancer-specific metabolic pathway has provided an exceptional opportunity for the development of new chemotherapeutic agents (2). These newly developed agents uniquely target this pathway and have theoretical advantages, which include cancer specificity and low toxicity to normal cells (5). 3-Bromopyruvate (3-BrPA), a member of 
this new class of anticancer drugs, is a specific alkylating agent and a potent ATP inhibitor, which blocks glycolytic tumor metabolism (6). Malignant cells generate a large amount of ATP via glycolysis $(7,8)$ and 3-BrPA has been shown to interrupt the glycolytic metabolic pathway, leading to the death of the tumor cells through apoptosis (9) with almost no damage to the surrounding normal tissues. It has been experimentally used to target hexokinase/glycolysis and possesses chemical and structural properties that are very similar to the acid, lactic acid and glucose metabolic products of glycolysis. These similar properties enable 3-BrPA to be transported by the same vector mechanisms that transport glycolytic products, and once it gains access to the pathway, 3-BrPA penetrates tumor cells and initiates its therapeutic effect (10).

Mitochondria are double membrane-enclosed organelles that play an essential role in cellular metabolism, ATP generation, ROS production and regulation of cell proliferation and death (11). They are also the principal source of ROS via electron leakiness in the electron transport chain. Disruption of the mitochondrial membrane gradient leads to increased electron leakage and potentially toxic ROS levels. Mitochondrial ROS were previously studied to determine their role in the tumor necrosis factor (TNF) $\alpha$-induced necrotic death of L929 cells (12). ROS including singlet oxygen, superoxide, hydroxyl free radical, hydrogen peroxides and nitric oxide mainly generated from the mitochondria play a crucial role in cell death (13). Necrosis is considered a passive cell death process, which is disorderly, unregulated and irreversible as opposed to the orderly programmed manner of apoptosis. However, ongoing studies on the mechanisms of cell death has put forth a new concept of type III programmed cell necrosis, known as necroptosis, which is a basic cell death pathway (14).

Z-VAD-fmk is a synthetic broad-spectrum caspase inhibitor that inhibits apoptosis. The serine-threonine kinase receptorinteracting protein (RIP) 1 is targeted by necrostatin-1 (Nec-1) a specific inhibitor of necroptosis, which depends on RIP1/3 complex activation $(15,16)$. Necroptosis controls normal embryonic organic evolution, T-cell propagation and chronic intestinal inflammation (17). In addition, the discovery of Nec-1, a small molecule that inhibits non-apoptotic programmed cell death-inducing substances, has provided a useful pharmacological tool for the study of necroptosis. In the present study, we treated NPC cells with 3-BrPA in the presence of Nec-1 and z-VAD-FMK to observe the therapeutic effects. The association between necroptosis and mitochondrial dysfunction remains to be examined in NPC cells.

\section{Materials and methods}

Reagents and antibodies. 3-BrPA, 3-(4,5-dimethylthiazol2-yl)-2,5-diphenyltetrazolium bromide (MTT), Nec-1, and NAC were purchased from Sigma (St. Louis, MO, USA). Z-VAD-fmk was purchased from Calbiochem. Dihydroethidium (DHE), JC-1, 4',6-diamidino-2-phenylindole (DAPI) and propidium iodide (PI) assay kits were purchased from Biotechnology (Beijing, China). Dulbecco's modified Eagle's medium (DMEM) and fetal bovine serum (FBS) were obtained from Gibco (Carlsbad, CA, USA). B-cell lymphoma 2 (Bcl-2), Bcl-2-associated protein X (Bax) and myeloid cell leukemia 1 (Mcl-1) antibodies were purchased from Abcam (Cambridge, UK). Mouse monoclonal antibodies were obtained from Cell Signaling Technology (Beverly, MA, USA). Rabbit anti- $\beta$-actin antibodies were obtained from Santa Cruz Biotechnology (Santa Cruz, CA, USA).

Cell lines and cell culture. NPC HNE1 and CNE-2Z cells were obtained from the Shanghai Cell Bank (Shanghai, China). The cells were grown in Roswell Park Memorial Institute (RPMI)-1640 medium supplemented with $10 \%$ fetal calf serum, streptomycin $(100 \mathrm{U} / \mathrm{ml})$, penicillin $(10 \mathrm{U} / \mathrm{ml})$, and HEPES $(25 \mathrm{mM})$. They were maintained at $37^{\circ} \mathrm{C}$ in a $5 \%$ $\mathrm{CO}_{2}$ humidified atmosphere. The NPC cells were seeded at a density of 8,000 cells/well in a 96-well plate for $24 \mathrm{~h}$ and then treated with different concentrations of 3-BrPA. At 24, 48 and $72 \mathrm{~h}$, the cells were incubated with MTT [5 mg/ml in phosphate-buffered saline, (PBS)] for $4 \mathrm{~h}$ at $37^{\circ} \mathrm{C}$. The MTT solution was then replaced with $100 \mu \mathrm{l}$ of dimethyl sulfoxide (DMSO)/well and the absorbance was measured using a plate reader at a wavelength of $490 \mathrm{~nm}$.

PI staining. Prior to treatment with $3-\mathrm{BrPA}$, the cells ( $2 \times 10^{5}$ cells/well) were plated in each well of a 12 -well plate and allowed to reach exponential growth $(24 \mathrm{~h})$. The cells were then treated with increasing concentrations of 3-BrPA (40, 80 and $160 \mu \mathrm{M}$ ) for $24 \mathrm{~h}$, stained with PI and then evaluated using flow cytometry.

DAPI staining and quantification of apoptotic cells. A DAPI nuclear staining assay was performed to examine the effects of 3-BrPA on cancer cell apoptosis. The CNE-2Z and HNE1 cells were plated in 6 -well plates on glass slides $\left(2 \times 10^{5}\right.$ cells/well). Following treatment with 3-BrPA for $24 \mathrm{~h}$, the cells were fixed with immunostaining setting for $30 \mathrm{~min}$ at $4^{\circ} \mathrm{C}$, washed twice with PBS and then incubated with DAPI for $30 \mathrm{~min}$ at $4^{\circ} \mathrm{C}$ in the dark. The slides were then washed with PBS to remove the excess DAPI and the cell nuclei were observed under a laser confocal scanning microscope (LCSM, Olympus 1x71). The apoptosis rate of the NPC cells treated with 3-BrPA $(40,80$, and $160 \mu \mathrm{M}$ ) was determined by scoring the number of cells with nuclear phenotypic changes in microscopic fields under the LCSM.

Intracellular ATP measurements. The cells were plated in duplicate in 96-well culture plates and the cellular ATP levels were determined using the Cell Titer-Glo luminescent cell viability assay (Promega, Madison, WI, USA) according to the manufacturer's instructions. The luminescent levels were measured using a microplate reader (Varioskan Flash spectral scanning multimode reader, Thermo Fisher, Waltham MA, USA).

Mitochondrial membrane potential (MMP) analysis. MMP detection was performed using the JC-1 staining assay kit according to the manufacturer's instructions. Briefly, following treatment with 3-BrPA, the cells were incubated with $10 \mu \mathrm{M}$ JC-1 for $30 \mathrm{~min}$ at $37^{\circ} \mathrm{C}$ in the dark. Then, the fluorescence in the cells was detected using a fluorescence microscope (Olympus 1x71).

Detection of superoxide anion levels. The HNE1 and CNE-2Z cells were seeded at a density of $2 \times 10^{5}$ cells/well in a 6 -well 

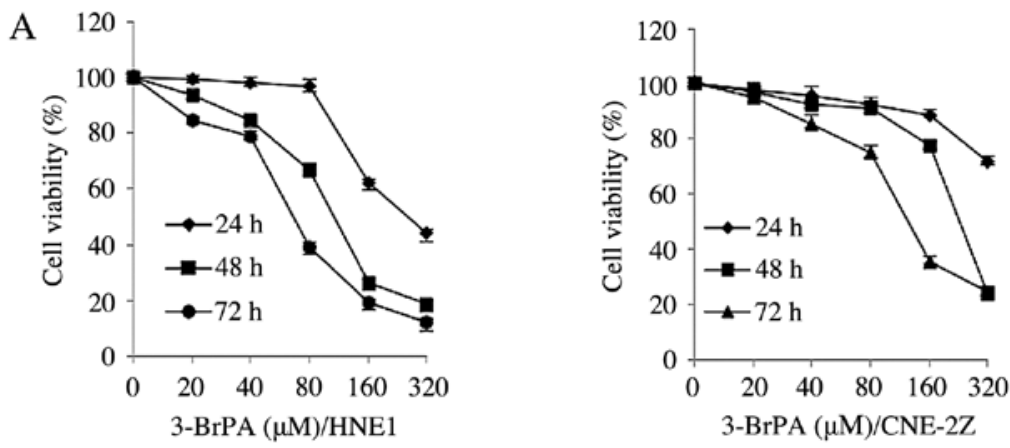

B

Control 20 40

80
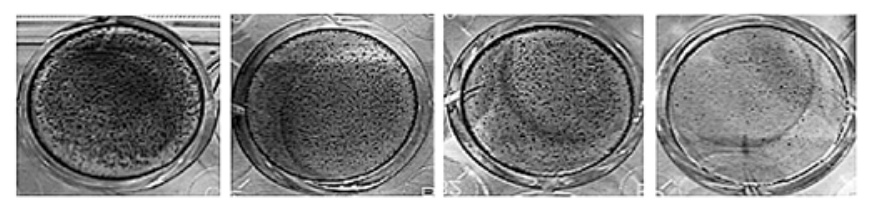

HNE1
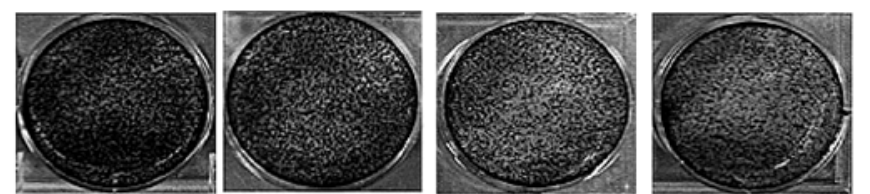

CNE-2Z

3-BrPA $(\mu \mathrm{M})$

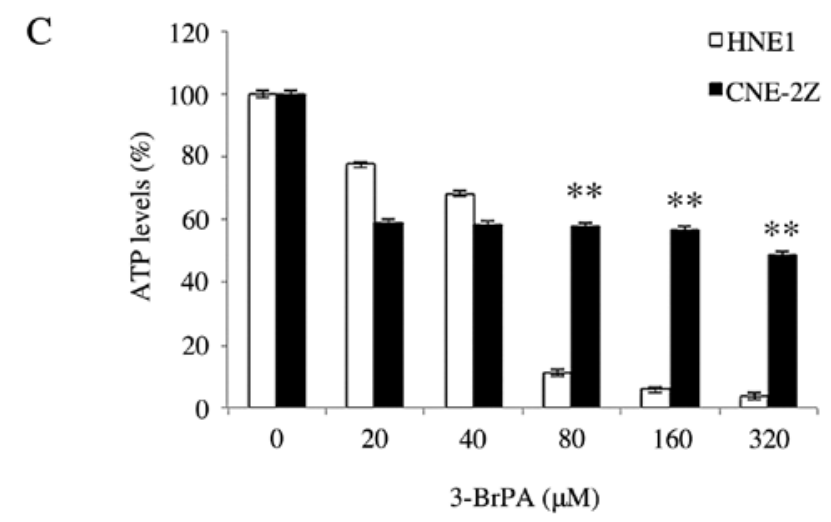

Figure 1. Inhibition of the proliferation of HNE1 and CNE-2Z cells by 3-BrPA. (A) HNE1 and CNE-2Z cells were treated with medium (Ctrl) and 20, 40, 80 , 160 and $320 \mu \mathrm{M}$ 3-BrPA for 24, 48 and $72 \mathrm{~h}$. Cell viability was measured using the MTT assay. (B) Inhibition of colony formation in HNE1 cells by 3-BrPA. HNE1 and CNE-2Z cells were treated with medium (Ctrl) and 20, 40 and $80 \mu \mathrm{M} 3$-BrPA for 5 days. (C) Reduction in ATP production and viability of malignant cells after 3-BrPA treatment. HNE1 and CNE-2Z cells were cultured with 40, 80, 160 and $320 \mu \mathrm{M} 3$-BrPA. Cellular levels of ATP were measured after 5 h. 3-BrPA, 3-bromopyruvate.

plate and labeled with $5 \mu \mathrm{M}$ DHE. This was immediately followed by treatment with 3 -BrPA $(160$ or $320 \mu \mathrm{M})$ for the indicated times and then the cells were collected, washed and analyzed using fluorescence-activated cell sorting (FACS).

Western blot analysis. The cells were rinsed with ice-cold PBS and lysed in radioimmunoprecipitation assay (RIPA) buffer for $30 \mathrm{~min}$ on ice, after which the lysates were centrifuged at $12,000 \mathrm{x} \mathrm{g}$ for $30 \mathrm{~min}$ at $4^{\circ} \mathrm{C}$. The proteins were then separated on a $15 \%$ sodium dodecyl sulfate (SDS)-polyacrylamide gel electrophoresis (PAGE) gel and subsequently transferred to a nitrocellulose membrane (Bio-Rad, Berkeley, CA, USA). The membranes were incubated with the appropriate primary antibodies overnight at $4^{\circ} \mathrm{C}$ and then further incubated with the corresponding secondary antibodies. $\beta$-actin was used as the loading control.
In vivo tumor experiment. The nude mice (3- to 4-weeks old) weighed $18-21 \mathrm{~g}$ at the time of tumor implantation. The mice were kept under a $12 / 12$-h light/dark cycle at $24 \pm 2^{\circ} \mathrm{C}$ and were given standard food and clean water. The mice were inoculated with the human CNE-2Z cells $\left(8 \times 10^{6}\right.$ cells $\left./ \mathrm{ml}\right)$ subcutaneously to induce tumor formation. Fifteen mice that developed tumors (100-200 $\left.\mathrm{mm}^{3}\right)$ were randomly assigned to three groups (5 mice/group). On day 7 after inoculation, the groups began to be treated intraperitoneally with vehicle [0.9\% normal saline, (NS)], 3-BrPA ( $8 \mathrm{mg} \cdot \mathrm{kg}^{-1}$ ) or cisplatin (DDP, $3 \mathrm{mg} \cdot \mathrm{kg}^{-1}$ ) treatment every 4 days for 28 days. Tumor growth was monitored every 4 days by obtaining two-dimensional measurements of the individual tumors of each mouse. The tumor volume was calculated using the formula: length $\mathrm{x}$ width ${ }^{2} / 2$. After the treatment ended, all the mice were euthanized and the tumors were excised. The tumor growth inhibition rate was calculated using 

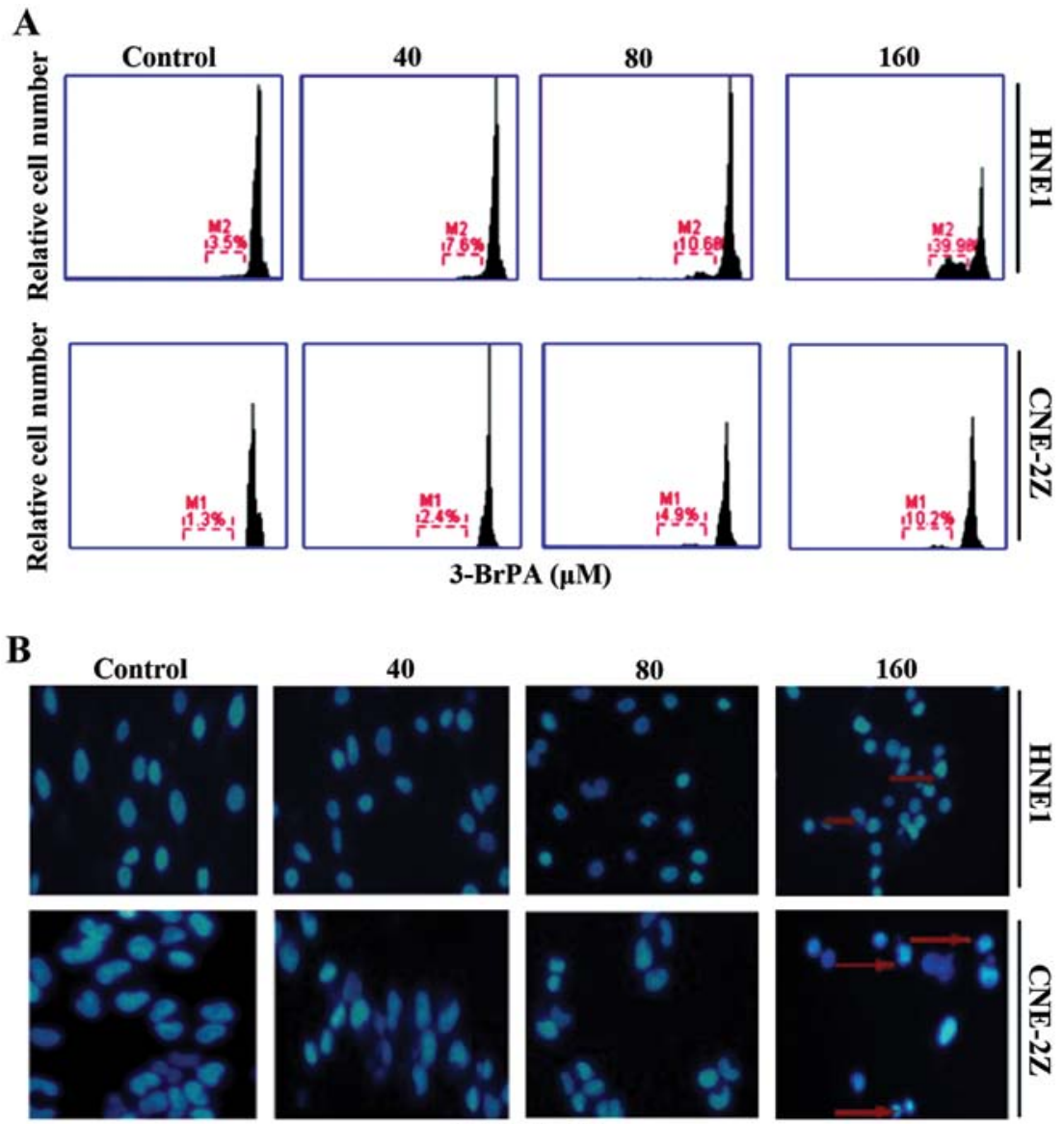

3-BrPA $(\mu \mathrm{M})$

Figure 2. 3-BrPA induces cell death in HNE1 cells. (A) HNE1 cells were treated with medium (Ctrl) and 40, 80 and $160 \mu \mathrm{M} 3-\mathrm{BrPA}$ for $24 \mathrm{~h}$. Cell death was measured by the PI staining method using flow cytometry. (B) NPC cells were treated with $0,40,80$, and $160 \mu \mathrm{M} 3$-BrPA for $24 \mathrm{~h}$, subjected to DAPI staining (showing nucleus) and then visualized under a fluorescence microscope. 3-BrPA, 3-bromopyruvate; PI, propidium iodide; NPC, nasopharyngeal carcinoma; DAPI, 4',6-diamidino-2-phenylindole.

the following formula: tumor inhibition rate $=(1-$ treatment group tumor weight/control group tumor weight) $\mathrm{x} 100 \%$. The tumors were then fixed in $4 \%$ formalin solution, embedded in paraffin and stained with hematoxylin and eosin (H\&E).

Statistical analysis. Data are expressed as the mean \pm SEM of three experiments. SPSS v.16.0 software (SPSS Inc., Chicago, IL, USA) was used for data analysis. The statistical analyses were carried out using one-way analysis of variance (ANOVA). $\mathrm{P}<0.05$ and $\mathrm{P}<0.01$ indicated values that were significantly different from the control values.

\section{Results}

Inhibition of NPC cell proliferation by varying concentrations of 3-BrPA. The effects of different concentrations of 3-BrPA were evaluated on the cell proliferation of two NPC cell lines treated for 24, 48 and $72 \mathrm{~h}$. We found that 3-BrPA inhibited proliferation and induced the apoptosis of HNE1 and CNE-2Z cells (Fig. 1A and B). The results demonstrated that the rate of apoptosis had a direct ratio relationship with the dose-response curve. Next, we determined the effects of 3-BrPA on the colony formation of the HNE1 and CNE-2Z cells by evaluating their 5-day survival rate in different concentrations of 3-BrPA. The results showed that the colony-forming ability of the cells was clearly reduced by 3-BrPA (Fig. 1B). The results show that the NPC cells with clonogenic capacity overcame the effects of glycolysis inhibition.

The role of glycolysis in ATP production in the HNE1 and CNE-2Z cells was evaluated by measuring the intracellular levels of ATP following treatment with the potent glycolysis inhibitor 3-BrPA for $5 \mathrm{~h}$. We found that the concentration of ATP decreased with increasing concentrations of 3-BrPA in both cell lines. However, the downward trend was more pronounced in the HNE1 cells. The results showed that 3-BrPA inhibits ATP production in the NPC cells (Fig. 1C).

3-BrPA induces apoptosis in NPC cells. In the MTT assay, we treated HNE1 and CNE-2Z cells with three different concentrations of 3-BrPA for $24 \mathrm{~h}$ and then used PI staining and flow cytometry to distinguish the apoptotic cells. The results showed that 3-BrPA induced apoptosis in the human HNE1 and CNE-2Z cells and the rate of apoptosis induction increased in a concentration-dependent manner (Fig. 2A). Many alkylating agents used to treat tumors act by inducing apoptosis (18) and therefore, we sought to determine whether 3-BrPA inhibited NPC cell proliferation by inducing apoptosis. The CNE-2Z and HNE1 cells were treated with different concentrations of 3-BrPA for $24 \mathrm{~h}$ and DAPI staining was used to detect apoptotic cells. The results showed that the 3-BrPA-treated 
A
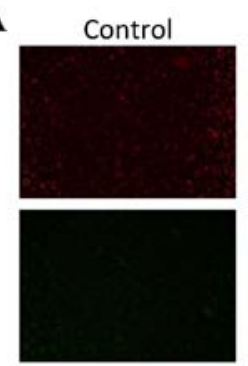

Control
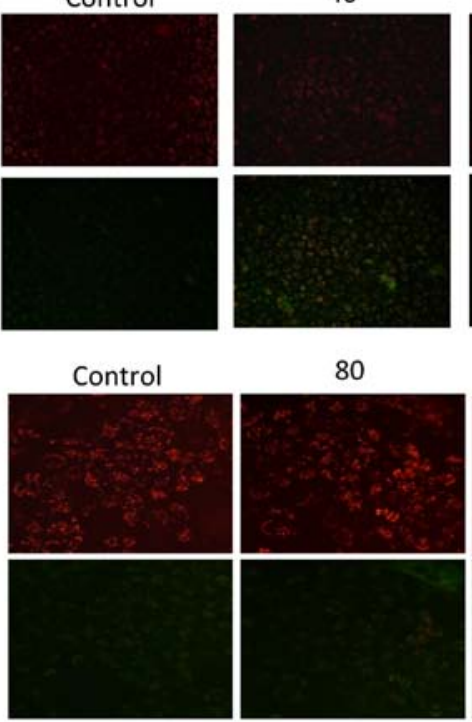

80

3-BrPA $(\mu \mathrm{M})$

B

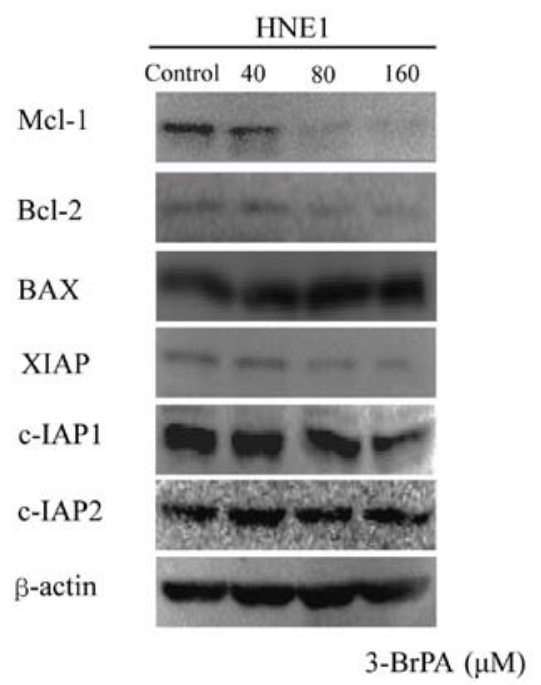

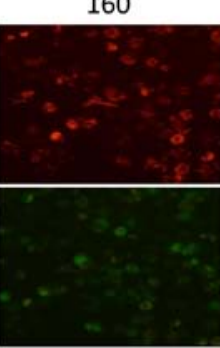

80
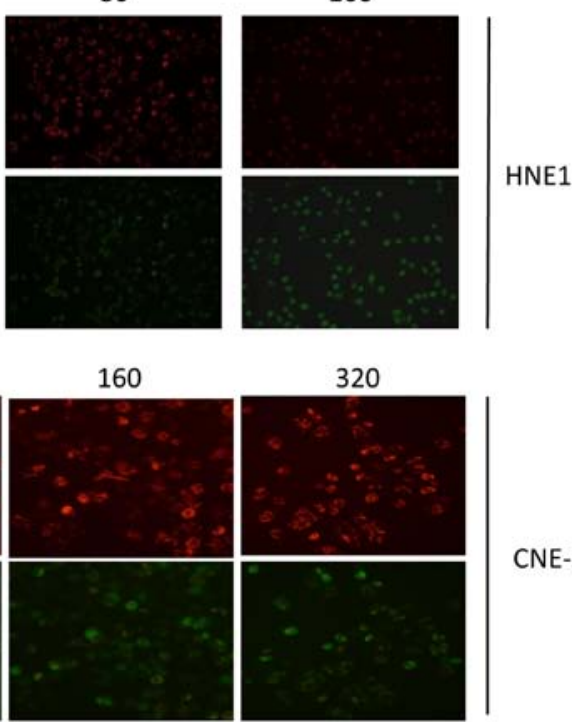

CNE-2Z

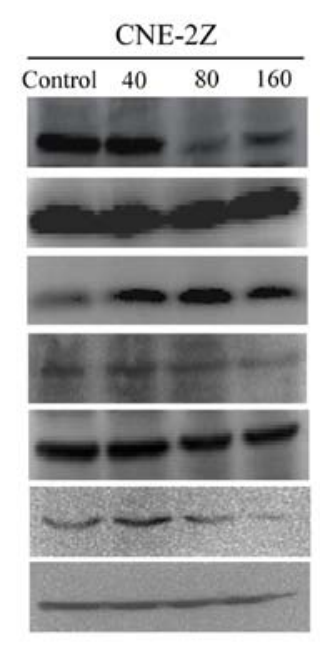

Figure 3. 3-BrPA reduces mitochondrial membrane potential (MMP, $\Delta \Psi \mathrm{m}$ ). (A) HNE1 cells were treated with 3-BrPA for 24 h and $\Delta \Psi \mathrm{m}$ was measured using JC-1 staining and observed under a fluorescence microscope with a camera. (B) The expression levels of apoptosis-related protein were determined by western blot analysis. NPC cells were treated with $0,40,80$ and $160 \mu \mathrm{M} 3$-BrPA for $24 \mathrm{~h}$; then, cell lysates were prepared and examined simultaneously using western blot analysis, n=3. 3-BrPA, 3-bromopyruvate; NPC, nasopharyngeal carcinoma.

HNE1 and CNE-2Z cells exhibited apoptotic characteristics of chromatin condensation with typical apoptotic bodies and the number of apoptotic nuclei was significantly increased in these groups (Fig. 2B).

3-BrPA reduces MMP and protein expression in NPC cells. JC-1 is a carbocyanine fluorescent probe widely used to detect MMP fluorescence, which we used to explore the mechanisms by which 3-BrPA induced apoptotic cell death in HNE1 and CNE-2Z cells. The normal mitochondria of the untreated control cells were characterized by red fluorescence while the depolarized mitochondria of the 3-BrPA-treated cells showed concentration-dependent, characteristic diffused green fluorescence. This result indicates that 3-BrPA increases mitochondrial membrane permeability (Fig. 3A). Although apoptosis may be induced by death receptors, the mitochondrial pathway is the major mediator of apoptosis and the induction of major outer-membrane proteins is essential for the initiation of this process. We sought to determine the mechanisms by which 3-BrPA induced apoptosis by measuring its effects on the expression of major outer-membrane proteins by using western blot analysis. The results showed that 3-BrPA inhibited the expression of the antiapoptotic proteins $\mathrm{Mcl}-1$ and $\mathrm{Bcl}-2$ in a dose-dependent manner and also the expression of the pro-apoptotic protein Bax. These results suggest that 3-BrPA may regulate the expression of apoptosis-related proteins. The expression of the inhibitors of apoptosis proteins (IAPs) including c-IAP1, c-IAP2, and XIAP was decreased by 3-BrPA in a dose-dependent manner, which also promoted the apoptosis of NPC cells (Fig. 3B).

Reactive oxygen species (ROS) production is involved in 3-BrPA-induced cell death. Next, we investigated the role of mitochondrial ROS activity in 3-BrPA-induced necroptosis. 

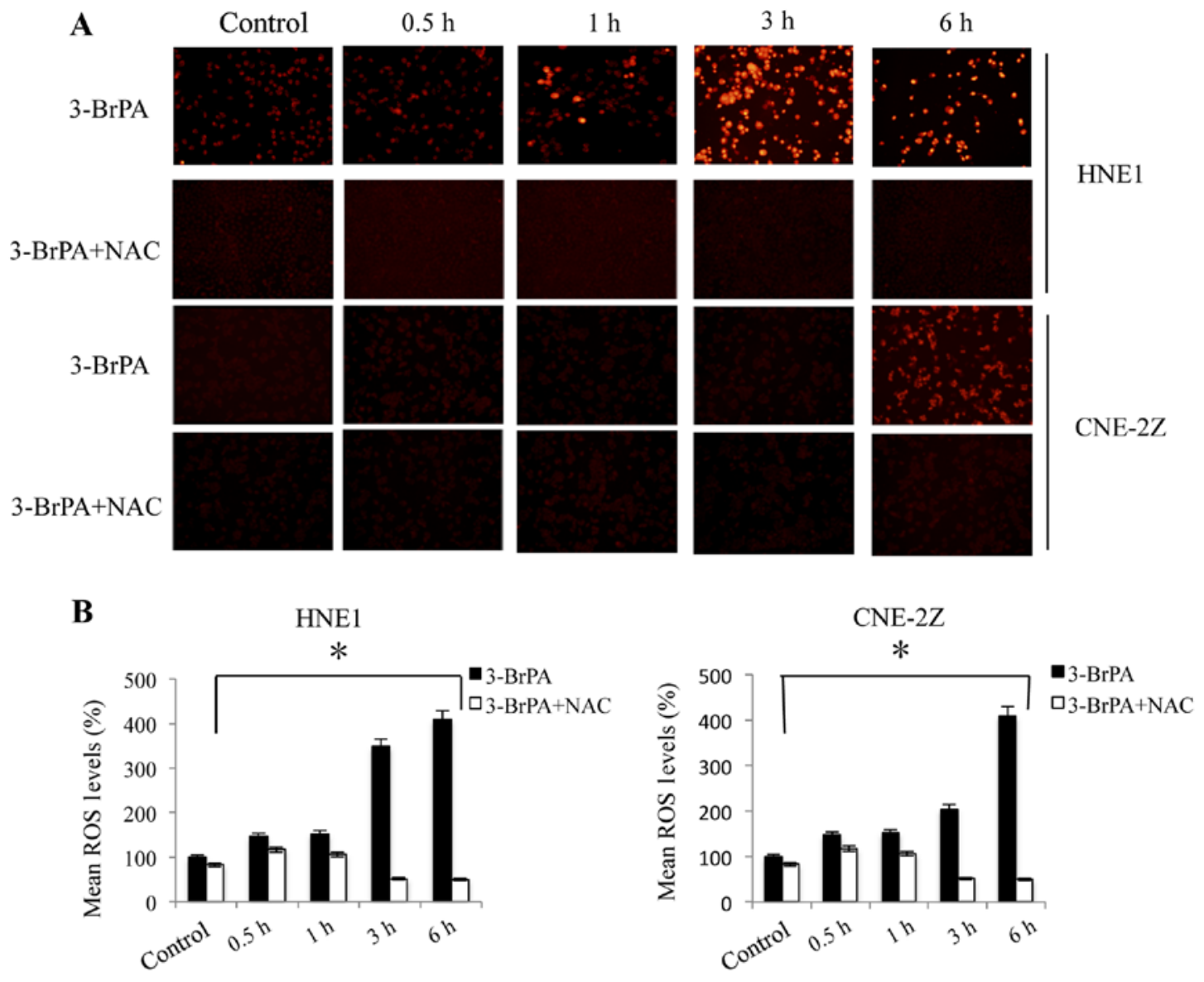

C

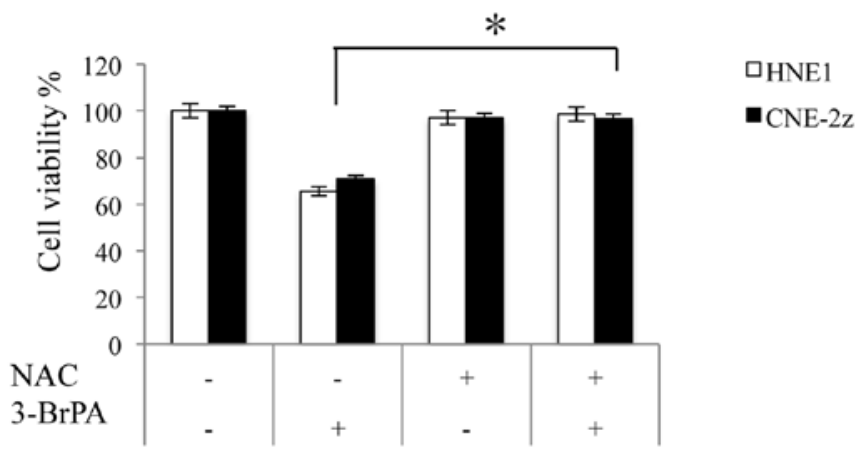

Figure 4. ROS production contributes to 3-BrPA-induced cell death in NPC cells. (A) HNE1 and CNE-2Z cells were treated with 160 and $320 \mu \mathrm{M} 3$-BrPA, respectively, with or without $5 \mathrm{mM} \mathrm{NAC}$ for $0.5,1,3$ and $6 \mathrm{~h}$. Cells were photographed using a fluorescence microscope camera. (B) After pre-incubation with $5 \mathrm{mM}$ NAC for $1 \mathrm{~h}$, cells were treated as above. ROS production was analyzed using DHE staining and flow cytometry. Mean ROS (100\%) values are shown. ${ }^{*} \mathrm{P}<0.05$ vs. the control at a given time-point. (C) After pre-incubation with $5 \mathrm{mM} \mathrm{NAC}$ for $1 \mathrm{~h}, \mathrm{HNE} 1$ and CNE- $2 \mathrm{Z}$ cells were treated with 160 and $320 \mu \mathrm{M}$ 3 -BrPA, respectively, for $24 \mathrm{~h}$ and the relative cell viability was assessed using the MTT assay. "P<0.05. Experiments were performed in triplicate. ROS, reactive oxygen species; 3-BrPA, 3-bromopyruvate; NPC, nasopharyngeal carcinoma; NAC, N-acetyl-L-cysteine; DHE, dihydroethidium.

We examined the effects of 3-BrPA on intracellular ROS production by using DHE, a fluorescent probe. As shown in Fig. 4A, cells treated with 3-BrPA showed a higher increase in the fluorescence intensity than did the control group $(\mathrm{P}<0.05)$. In addition, the fluorescence intensity of the cells treated with the antioxidant $\mathrm{N}$-acetyl-L-cysteine (NAC, $5 \mathrm{mM}$ ) alone was reduced more than that of the cells treated with 3-BrPA alone. Furthermore, the DHE assay revealed high levels of green fluorescence in cells co-treated with 3-BrPA and NAC, while the addition of more NAC darkened the cells again (Fig. 4A). In addition, we discovered that 3-BrPA increased the ROS levels in a time-dependent manner within $6 \mathrm{~h}$ (Fig. 4B). These results showed that NAC $(5 \mathrm{mM})$ completely blocked the effect of 3-BrPA and reduced the intracellular ROS levels (Fig. 4B). Finally, the viability of the NPC cells treated with 160 or $320 \mu \mathrm{M} 3$-BrPA was decreased significantly, and this effect was blocked by 5 mM NAC (Fig. 4C).

3-BrPA induces cell death in NPC cells by necroptosis. To confirm the type of cell death induced in HNE1 and CNE-2Z cells by 3 -BrPA, we evaluated the inhibitory effects of z-VAD-fmk, a broad-spectrum caspase inhibitor, on 3-BrPA. The MTT assay (Fig. 5A) showed that z-VAD-fmk aggravated cell death in the HNE1 and CNE-2Z cells. The cell 


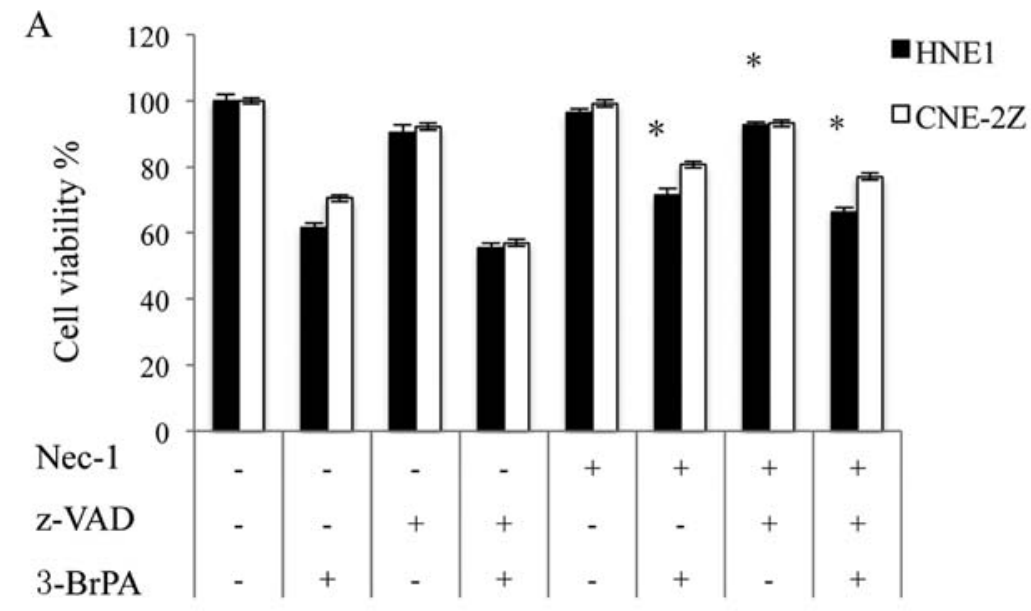

B
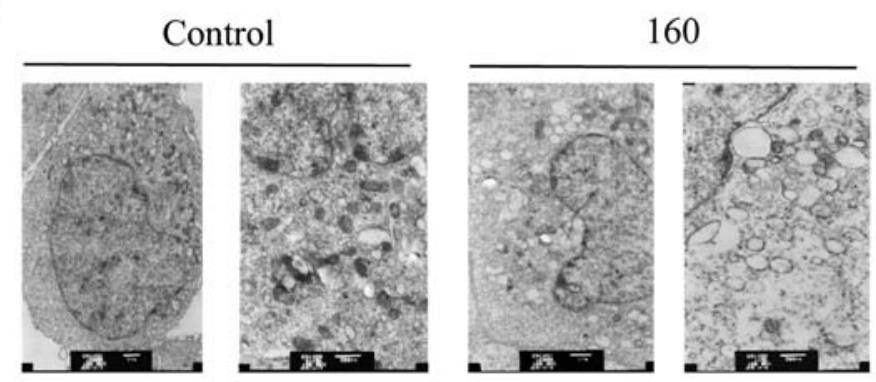

HNE1

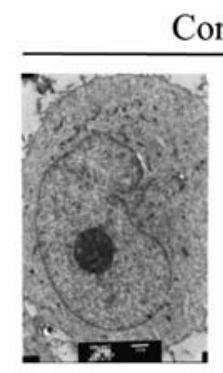

Control
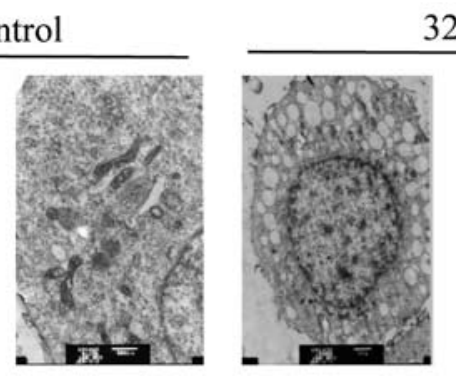

320

\section{3-BrPA $(\mu \mathrm{M})$}

Figure 5. 3-BrPA plus z-VAD-fmk induce necroptosis in human NPC cells. (A) HNE1 and CNE-2Z cells were treated with medium (Ctrl), and 160 and $320 \mu \mathrm{M}$ 3-BrPA with $20 \mu \mathrm{M}$ z-VAD-fmk or $20 \mu \mathrm{M} \mathrm{Nec}-1$ for $24 \mathrm{~h}$. Other cells were treated with $20 \mu \mathrm{M}$ z-VAD-fmk or Nec-1 alone. Cell viability was determined using the MTT assay. (B) HNE1 and CNE-2Z cells were treated with 160 and $320 \mu \mathrm{M} 3-\mathrm{BrPA}$ for $24 \mathrm{~h}$ and then the cells were collected and prepared for EM analysis. Scale bar, $1 \mu \mathrm{m}$; Ctrl, control; 3-BrPA, 3-bromopyruvate; NPC, nasopharyngeal carcinoma; Nec-1, necrostatin-1; EM, electron microscopy.

death observed was possibly mediated by necroptosis, the programmed form of necrosis, which involves RIP1 and RIP3 triggering the death receptors (19). Nec-1 alone had no effect on both HNE1 and CNE-2Z cells, but radically restored cell survival of 3-BrPA-treated cells (Fig. 5A). Electron microscopy (EM) showed that the 3-BrPA-treated HNE1 and CNE-2Z cells had ruptured plasma membranes, which is indicative of necrosis (Fig. 5B).

In vivo antitumor efficacy of 3-BrPA. We investigated the in vivo antitumor activity of 3 -BrPA using tumor-bearing nude mice. On day 7 after inoculation, mice bearing CNE-2Z tumors were intraperitoneally administere $3-\mathrm{BrPA}\left(8 \mathrm{mg} \cdot \mathrm{kg}^{-1}\right)$ and DDP $\left(3 \mathrm{mg} \cdot \mathrm{kg}^{-1}\right)$ treatment every 4 for 28 days. The tumor volumes of the treated mice increased at a much slower rate than those of the control group (Fig. 6A and B). The tumors were completely excised 35 days after the cancer cell inoculation. The tumor weight and growth inhibition rate for the three groups are shown in Fig. 6C. The transplanted tumor tissue sections from all three groups showed poorly differentiated squamous cancer cells with varying degrees of pathological mitosis and eosinophilic necrosis. The DDP and 3-BrPA-treated CNE-2Z tumors showed a consistent size and shape, and the cell size was smaller than that of the untreated control cells. These tumors also showed a decreased nuclear-cytoplasmic ratio. In addition, the number of nucleoli, pathological mitosis and number of vessels were lower in the treated tumors than these in the tumors in the control group, while fibrous tissue increased owing to eosinophilic necrosis (Fig. 6D).

\section{Discussion}

There are currently several treatment options for NPC, including radiotherapy, chemotherapy, surgery and chemotherapy. However, none of them provides an ideal or optimal therapeutic effect. The commonly used chemotherapeutic 
A

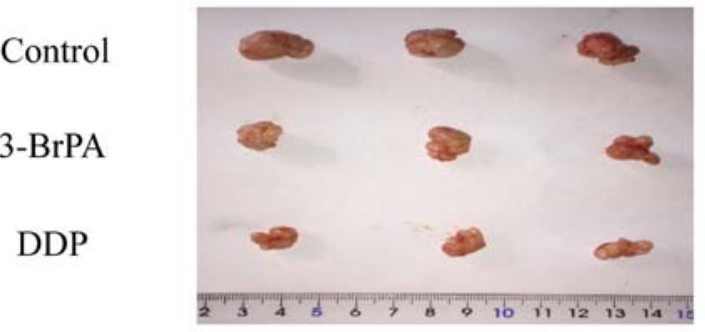

B

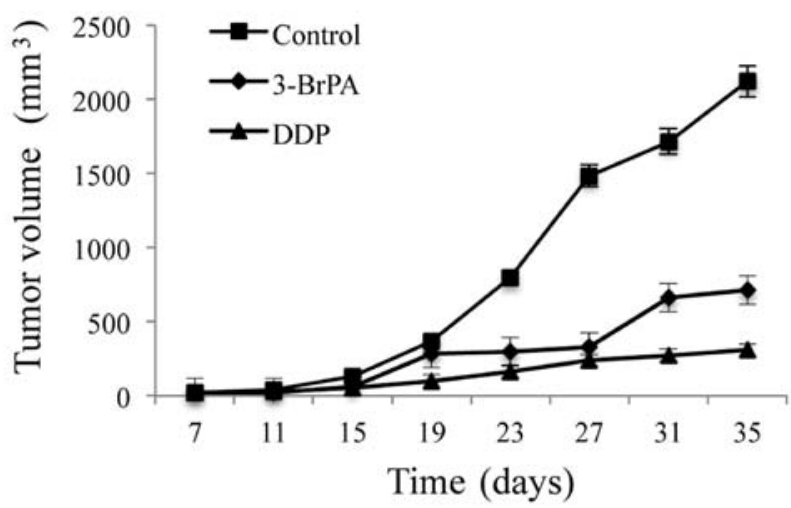

$\mathrm{C}$

The weight of engraft in different groups $(\mathrm{x} \pm \mathrm{s})$

\begin{tabular}{ccc}
\hline Groups & Tumor weight $(\mathrm{g})$ & Tumor suppression rate \\
\hline Control & $1.7 \pm 0.37$ & -- \\
3-BrPA & $0.78 \pm 0.13^{*}$ & $54.12 \%$ \\
DDP & $0.34 \pm 0.15^{*}$ & $80 \%$ \\
\hline
\end{tabular}

$\mathrm{D}$

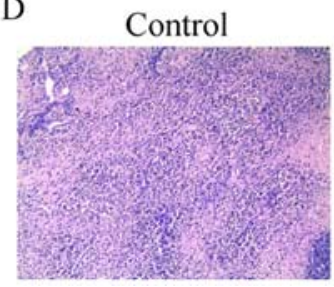

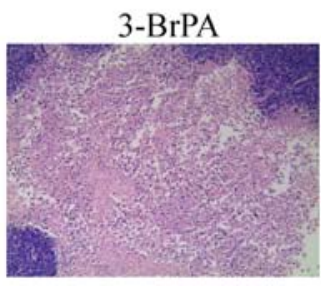

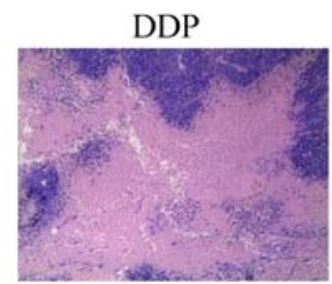

Figure 6. Antitumor activity of 3-BrPA in nude mice. CNE-2Z cells were inoculated subcutaneously to form tumors. Mice with tumors $\left(100-200\right.$ mm $\left.{ }^{3}\right)$ were randomized into three groups (5 mice/group). On day 7 after inoculation, vehicle $\left(0.9 \%\right.$ normal saline), cisplatin (DDP, 3 mg $\left.\cdot \mathrm{kg}^{-1}\right)$ or 3 -BrPA $\left(8 \mathrm{mg}^{\left.-\mathrm{kg}^{-1}\right)} \mathrm{was}\right.$ administered to the mice intraperitoneally every 4 days. (A) Comparison of the transplanted tumors. (B) Tumor growth was monitored every 4 days and calculated using the formula: length $\mathrm{x}$ width ${ }^{2} / 2$. (C) Tumor weights and tumor growth inhibition rate after 3-BrPA and DDP treatment. (D) H\&E staining in vivo. 3-BrPA, 3-bromopyruvate; $\mathrm{H} \& \mathrm{E}$, hematoxylin and eosin.

drugs are highly toxic and susceptible to drug resistance. Thus, strategies for reducing the toxic side effects of the drugs while simultaneously improving drug efficacy are an important aspect of anticancer drug development (20).

3-BrPA has recently become a popular drug among research scholars worldwide (21). This drug is a glycolytic inhibitor that acts via hexokinase II and glyceraldehyde-3-phosphate dehydrogenase (GAPDH) and plays a critical role in apoptosis induced by intracellular ATP depletion. At the same time, it also inhibits succinate dehydrogenase (SDH) activity, which causes an imbalance in the cellular redox state. The additional inhibition of the hexokinase II pentose phosphate pathway leads to a decrease in the activity of the NADPH-generating pentose phosphate pathway (PPP), ultimately reducing the equivalent intracellular NADPH levels and further decreasing the peroxide state. Therefore, 3-BrPA lowers the levels of intracellular ATP and induces an imbalance in the intracellular oxidative metabolism, with good antitumor effects $(22,23)$. Our results showed that 3-BrPA inhibited NPC HNE1 and CNE-2Z cell proliferation, triggered apoptosis, reduced ATP production and increased intracellular ROS levels.

Bcl-2 and Bax exist as either homologous dimers or heterodimers and form an apoptosis regulatory system in combination with the B-cell lymphoma-extra-large (Bcl-xL) protein. The formation of Bax homologous dimers induces apoptosis while $\mathrm{Bcl}-2$ protein expression causes the separation of these dimers, thereby increasing the Bcl-2 to Bax-Bax ratio. This increase in Bcl-2 facilitates the creation of the more stable Bax-Bcl-2 heterodimer, thereby 'neutralizing' the Bax-Bax dimer-induced apoptosis. Thus, the Bcl-2 and Bax proportion controls apoptosis $(24,25)$. Our results revealed a decrease in the expression of Bcl-2 in HNE-1 cells with an increase in Bax 
expression. However, in the $\mathrm{CNE}-2 \mathrm{Z}$ cells, the $\mathrm{Bcl}-2$ protein expression remained essentially unchanged while BAX expression increased. Members of the $\mathrm{Bcl}-2$ protein family can induce imbalances in the endogenous pathway regulating apoptosis (26). The IAP-induced direct inhibition of caspases or pro-caspases mainly suppresses key members of the caspase family. Thus, IAPs such as c-IAP1, c-IAP2, XIAP, livin and survivin proteins directly inhibit caspases 3, 7 and 9. The IAPs possess a highly conserved BIR and RING domain structure, which interacts with the protease caspases and plays an antiapoptotic role by inhibiting caspase activity (25).

Many studies have shown that ROS such as peroxide induce apoptosis in many different cell types including neutrophils (gas apoptosis) and this process is mediated by catalase inhibition (27). The mercapto group donor in NAC confers antioxidant properties by clearing the generated free groups, thereby regulating cellular metabolic activity $(28,29)$. In contrast, 3-BrPA facilitates an increase in intracellular ROS levels and has been widely used in clinical studies because of this activity. The actions of NAC and 3-BrPA render them highly useful joint pharmacological tools. While NAC protects the cells, 3-BrPA enhances the intracellular ROS content, which induces apoptosis by regulating cell death. The resultant apoptotic cell death model is well known and widely used.

The pathway of essential cell death is invoked by various cellular stresses, including DNA damage, oxidative stress, growth factor deprivation, heat shock and endoplasmic reticulum stress (30). With the passage of time, experimental advances and discoveries, researchers are now more interested in the concept of necrotic cell death. Necrotic death generally occurs as a result of high-dose cytotoxic abuse and does not require any specific molecular programming. However, it is now recognized that mechanisms other than those mediated by caspase induce cellular suppression through a caspase-independent pathway of cell death that exhibits the morphological characteristics of necrosis. This form of regulated cell death was recently termed 'necrotizing apoptosis' or 'necroptosis' (14), because it shares features of both apoptosis and necrosis. As in the case of apoptosis, necroptosis involves an explicit molecular cascade. However, both necroptosis and necrosis are characterized by swelling of the cell and its organelles and cell rupture, which leads to the release of cellular contents (31). One example of necroptosis can be observed in HT-29 cells, wherein z-VAD-fmk accelerates Smac analog-induced cell death, which is inhibited by Nec-1, the necrosis-specific chemical inhibitor (32). We also discovered that Nec-1 protects HNE1 and CNE-2Z cells against the acceleration of 3-BrPA-induced cell death via z-VAD-fmk. However, additional experiments are required to confirm how 3-BrPA induces necroptosis in HNE1 and CNE-2Z cells treated with caspase inhibitors. Necroptosis and apoptosis are two different forms of death receptor-mediated cell death. These processes are caspase-independent and do not require mitochondrial release of cytochrome $c$, but are controlled by a signal-regulated process mediated by RIP1/RIP3. Inhibition of caspase activity in cells will inhibit apoptosis but not necrosis. Nec-1 acts as a specific inhibitor of programmed necrosis by inhibiting the formation of the RIP1-RIP3 complex; therefore, the inhibition of RIP1 interferes with necroptosis (33).
In conclusion, the results of the present study showed that 3-BrPA significantly inhibits the proliferation of human NPC HNE1 and CNE-2Z cells and induces apoptosis by increasing ROS levels. In addition, the mechanism of action of 3-BrPA may be mediated via the mitochondrial pathway in cells and the death-receptor pathway. These results suggest that 3-BrPA may be a potential candidate for development as a new anticancer drug. However, the instability of 3-BrPA in solution poses a serious challenge to developing a safe and stable potent inhibitor of hexokinase, which is certain to become an important research topic.

\section{Acknowledgements}

The present study was supported by grants from the National Natural Science Foundation of China (81000992 and 81372899), the Natural Science Foundation of the Anhui Province (1508085MH166), the Graduate Scientific Research and Innovation Projects of Bengbu Medical College of Anhui Province (Byycx1421) and the Science Foundation of Bengbu Medical College (Byky1447).

\section{References}

1. Hildesheim A and Levine PH: Etiology of nasopharyngeal carcinoma: A review. Epidemiol Rev 15: 466-485, 1993.

2. Geschwind JF, Ko YH, Torbenson MS, Magee C and Pedersen PL: Novel therapy for liver cancer: Direct intraarterial injection of a potent inhibitor of ATP production. Cancer Res 62: 3909-3913, 2002.

3. Warburg O: On the origin of cancer cells. Science 123: 309-314, 1956.

4. Nelson K: 3-Bromopyruvate kills cancer cells in animals. Lancet Oncol 3: 524, 2002.

5. Finley RS: Overview of targeted therapies for cancer. Am J Health Syst Pharm 60: S4-S10, 2003.

6. Mathupala SP, Ko YH and Pedersen PL: Hexokinase-2 bound to mitochondria: Cancer's stygian link to the 'Warburg Effect' and a pivotal target for effective therapy. Semin Cancer Biol 19: 17-24, 2009.

7. Buchakjian MR and Kornbluth S: The engine driving the ship: Metabolic steering of cell proliferation and death. Nat Rev Mol Cell Biol 11: 715-727, 2010.

8. Kondoh H: Cellular life span and the Warburg effect. Exp Cell Res 314: 1923-1928, 2008.

9. Nakano A, Tsuji D, Miki H, Cui Q, El Sayed SM, Ikegame A, Oda A, Amou H, Nakamura S, Harada T, et al: Glycolysis inhibition inactivates $\mathrm{ABC}$ transporters to restore drug sensitivity in malignant cells. PLoS One 6: e27222, 2011.

10. Dolmans DE, Kadambi A, Hill JS, Flores KR, Gerber JN, Walker JP, Borel Rinkes IH, Jain RK and Fukumura D: Targeting tumor vasculature and cancer cells in orthotopic breast tumor by fractionated photosensitizer dosing photodynamic therapy. Cancer Res 62: 4289-4294, 2002.

11. Peter ME: Programmed cell death: Apoptosis meets necrosis. Nature 471: 310-312, 2011.

12. Goossens V, Stange G, Moens K, Pipeleers D and Grooten J: Regulation of tumor necrosis factor-induced, mitochondria- and reactive oxygen species-dependent cell death by the electron flux through the electron transport chain complex I. Antioxid Redox Signal 1: 285-295, 1999.

13. Trachootham D, Lu W, Ogasawara MA, Nilsa RD and Huang P: Redox regulation of cell survival. Antioxid Redox Signal 10: 1343-1374, 2008.

14. Degterev A, Huang Z, Boyce M, Li Y, Jagtap P, Mizushima N, Cuny GD, Mitchison TJ, Moskowitz MA and Yuan J: Chemical inhibitor of nonapoptotic cell death with therapeutic potential for ischemic brain injury. Nat Chem Biol 1: 112-119, 2005.

15. Declercq W, Vanden Berghe T and Vandenabeele P: RIP kinases at the crossroads of cell death and survival. Cell 138: 229-232, 2009.

16. Degterev A, Zhou W, Maki JL and Yuan J: Assays for necroptosis and activity of RIP kinases. Methods Enzymol 545: 1-33, 2014. 
17. Humphries F, Yang S, Wang B and Moynagh PN: RIP kinases: Key decision makers in cell death and innate immunity. Cell Death Differ 22: 225-236, 2015.

18. Parlato M, Souza-Fonseca-Guimaraes F, Philippart F, Misset B, Adib-Conquy M and Cavaillon JM; Captain Study Group: CD24-triggered caspase-dependent apoptosis via mitochondrial membrane depolarization and reactive oxygen species production of human neutrophils is impaired in sepsis. J Immunol 192: 2449-2459, 2014.

19. Galluzzi L and Kroemer G: Necroptosis: A specialized pathway of programmed necrosis. Cell 135: 1161-1163, 2008.

20. Ahmad S and Ansari AA: Therapeutic roles of heparin anticoagulants in cancer and related disorders. Med Chem 7: 504-517, 2011.

21. Shoshan MC: 3-Bromopyruvate: Targets and outcomes. J Bioenerg Biomembr 44: 7-15, 2012.

22. Cardaci S, Desideri E and Ciriolo MR: Targeting aerobic glycolysis: 3-bromopyruvate as a promising anticancer drug. J Bioenerg Biomembr 44: 17-29, 2012.

23. Dell'Antone P: Targets of 3-bromopyruvate, a new, energy depleting, anticancer agent. Med Chem 5: 491-496, 2009.

24. Scarfò L and Ghia P: Reprogramming cell death: BCL2 family inhibition in hematological malignancies. Immunol Lett 155 : 36-39, 2013.

25. Ndubaku C, Varfolomeev E, Wang L, Zobel K, Lau K, Elliott LO, Maurer B, Fedorova AV, Dynek JN, Koehler M, et al: Antagonism of c-IAP and XIAP proteins is required for efficient induction of cell death by small-molecule IAP antagonists. ACS Chem Biol 4: $557-566,2009$.
26. Rossé T, Olivier R, Monney L, Rager M, Conus S, Fellay I, Jansen B and Borner C: Bcl-2 prolongs cell survival after Bax-induced release of cytochrome c. Nature 391: 496-499, 1998.

27. Kasahara Y, Iwai K, Yachie A, Ohta K, Konno A, Seki H, Miyawaki $T$ and Taniguchi N: Involvement of reactive oxygen intermediates in spontaneous and CD95 (Fas/APO-1)-mediated apoptosis of neutrophils. Blood 89: 1748-1753, 1997.

28. De Flora S, Izzotti A, D'Agostini F and Balansky RM: Mechanisms of $\mathrm{N}$-acetylcysteine in the prevention of DNA damage and cancer, with special reference to smoking-related end-points. Carcinogenesis 22: 999-1013, 2001.

29. Wang CC, Liu TY, Cheng CH and Jan TR: Involvement of the mitochondrion-dependent pathway and oxidative stress in the apoptosis of murine splenocytes induced by areca nut extract. Toxicol In Vitro 23: 840-847, 2009.

30. de Almagro MC and Vucic D: Necroptosis: Pathway diversity and characteristics. Semin Cell Dev Biol 39: 56-62, 2015.

31. Kaczmarek A, Vandenabeele P and Krysko DV: Necroptosis: The release of damage-associated molecular patterns and its physiological relevance. Immunity 38: 209-223, 2013.

32. He S, Wang L, Miao L, Wang T, Du F, Zhao L and Wang X: Receptor interacting protein kinase-3 determines cellular necrotic response to TNF-alpha. Cell 137: 1100-1111, 2009.

33. Xu X, Chua CC, Kong J, Kostrzewa RM, Kumaraguru U, Hamdy RC and Chua BH: Necrostatin-1 protects against glutamate-induced glutathione depletion and caspase-independent cell death in HT-22 cells. J Neurochem 103: 2004-2014, 2007. 